\title{
Determination of Colistin Resistance by Simple Disk Diffusion Test Using Modified Mueller-Hinton Agar
}

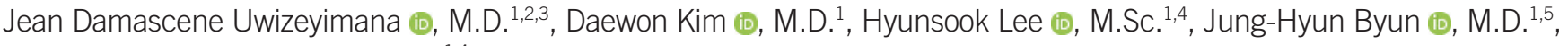 \\ and Dongeun Yong $\mathbb{0}$, M.D., Ph.D. ${ }^{1,4}$ \\ ${ }^{1}$ Department of Laboratory Medicine and Research Institute of Bacterial Resistance, Yonsei University College of Medicine, Seoul, Korea; ${ }^{2}$ Department of \\ Global Health Security, Yonsei University Graduate School of Public Health, Seoul, Korea; ${ }^{3}$ Department of Emergency Care, Ruli Hospital, Gakenye, Rwanda; \\ ${ }^{4}$ Brain Korea 21 plus Program for Medical Science, Yonsei University College of Medicine, Seoul, Korea; ${ }^{5}$ Department of Laboratory Medicine, Gyeongsang \\ National University Hospital, Gyeongsang National University College of Medicine, Jinju, Korea
}

Background: Colistin has become a last-resort antibiotic for the management of multidrugresistant gram-negative bacteria. The disk diffusion test is cheap and easy to perform but may be unreliable for colistin susceptibility testing due to poor diffusion of the large colistin molecule. An improved agar diffusion test would increase the reliability of colistin susceptibility testing. This study aimed to modify Muller-Hinton agar (MHA) to improve colistin diffusion in agar.

Methods: MHA was modified by reducing the agar concentration from 100\% to $30 \%$ and supplementing with protamine. We tested 60 gram-negative clinical isolates of Pseudomonas aeruginosa $(\mathrm{N}=27)$ and Acinetobacter calcoaceticus-baumannii complex $(\mathrm{N}=33)$. Disk diffusion test results were interpreted based on minimum inhibitory concentrations determined by broth microdilution.

Results: The modified MHA yielded the best performance metrics, including 94.7\% sensitivity, 100\% specificity, and an area under the curve of 0.995 (95\% confidence interval, 0.982-1.000), $P<0.001$, at a cut-off point of $13 \mathrm{~mm}$.

Conclusions: A reduction of the agar concentration from 100\% to 30\% and the addition of protamine improved colistin diffusion in agar and allowed routine colistin susceptibility testing in a clinical microbiology laboratory, but should be handled with caution.

Key Words: Colistin, Disk diffusion, Colistin susceptibility testing, Muller-Hinton agar, Protamine
Received: June 3, 2019

Revision received: October 8, 2019

Accepted: February 7, 2020

\section{Corresponding author:}

Jung-Hyun Byun, M.D.

Department of Laboratory Medicine,

Gyeongsang National University Hospital, Gyeongsang National University College of Medicine, Jinju 52727, Korea

Tel: +82-55-750-8423

Fax: +82-55-762-2696.

E-mail: microbyun@gmail.com

\section{Co-corresponding author:}

Dongeun Yong, M.D., Ph.D.

Department of Laboratory Medicine and Research Institute of Bacterial Resistance, Yonsei University College of Medicine, 50 Yonsei-ro, Seodaemun-gu, Seoul

03722, Korea

Tel: +82-2-2228-2454

Fax: +82-2-364-1583.

E-mail: deyong@yuhs.ac

\section{(c) (i) (2)}

(c) Korean Society for Laboratory Medicine This is an Open Access article distributed under the terms of the Creative Commons Attribution Non-Commercial License (https://creativecommons.org/licenses/by-nc/4.0) which permits unrestricted non-commercial use, distribution, and reproduction in any medium, provided the original work is properly cited.

\section{INTRODUCTION}

With limited new antibiotic classes in the drug development pipeline, the global challenge of antimicrobial resistance, particularly in the treatment of multidrug-resistant (MDR) gram-nega- tive (GN) bacteria, remains critical. Colistin, also known as polymyxin $E$, has become a last-resort antibiotic for the management of MDR GN bacteria [1, 2]. Colistin, a member of an old class of cationic, cyclic, polypeptide antibiotics was first introduced in Japan in 1947 from the soil bacterium Paenibacillus 
polymyxa and adopted for clinical use in 1959 [3].

Although its use was abandoned in the 1980s because of concerns of nephrotoxicity and neurotoxicity [4], intravenous injection of colistin sulfate was demonstrated to be safe and could be considered for the management of severe infections from sensitive MDR GN pathogens [5]. Therefore, beginning in the mid-1990s, the clinical use of polymyxins was revived, with a focus on colistin because of its rapid bactericidal effect, broadspectrum activity against MDR GN pathogens, and the lack of novel antibiotics against the most prevalent MDR GN bacteria [3, 5-10]. The global colistin resistance rate is less than $10 \%$, but is increasing. Increased resistance has been reported in Mediterranean and Southeast Asian countries [11].

The increasing use of colistin over the past several years has necessitated rapid, accurate, and reliable in-vitro antimicrobial susceptibility testing (AST) methods to allow appropriate therapeutic decisions. The disk diffusion test, commonly used in clinical laboratories, is considered to be unreliable because colistin diffuses poorly into agar due to electrostatic interactions with acid or sulfate groups of agar, resulting in smaller inhibition zones [12]. The poor diffusion results in high error rates compared to the broth microdilution (BMD) test for minimum inhibitory concentration (MIC) determination [13]. Other methods, such as the VITEK 2 AST system (bioMérieux, Marcy l'Etoile, France), Etest (AB Biodisk, Solna, Sweden), and agar dilution, also remain unreliable [14-19]. There is currently no standardized disk diffusion test for colistin susceptibility testing for use in clinical microbiology laboratories [20].

This study aimed to develop a simple disk diffusion test method for colistin susceptibility testing by modifying the commercial Mueller-Hinton medium to improve colistin diffusion in agar.

\section{MATERIALS AND METHODS}

\section{Phase I}

Disk diffusion test

The colistin disk diffusion test was performed using a $10 \mathrm{mg}$ colistin disk on Mueller-Hinton agar (MHA) plates that were incubated at $35^{\circ} \mathrm{C}$ for $16-18$ hours in $5 \% \mathrm{CO}_{2}$. Disk diffusion test results were interpreted based on the diameter of inhibition zone and compared with MICs determined by BMD according to the 2018 CLSI guidelines [21].

Optimization of the agar concentration

First, an optimum agar concentration was determined using four strains: P. aeruginosa ATCC 27853, a colistin-susceptible Aci- netobacter calcoaceticus-baumannii complex (ACB) strain, a colistin-resistant ACB strain, and mcr-1-harboring Klebsiella aerogenes. Assays were run in triplicate. Species were identified using a Microflex LT Biotyper (Bruker Daltonics, Leipzig, Germany). MHA was modified by reducing the agar granule concentration from $100 \%$ (17 g/L) to $30 \%(5.1 \mathrm{~g} / \mathrm{L})$ of the concentration in commercial MHA with 10\% intervals (Becton, Dickinson, \& Company, Sparks, MD, USA). The optimum concentration was determined based on the least agar concentration that was manageable in the laboratory. Lower the agar concentration, more fragile is the AST determination. The final agar concentration was reduced to $30 \%$.

Optimization of the protamine concentration

We added protamine (Sigma-Aldrich, St Louis, MO, USA) to the modified MHA at various concentrations $(1,000 \mu \mathrm{g} / \mathrm{mL}, 700 \mu \mathrm{g} /$ $\mathrm{mL}, 400 \mu \mathrm{g} / \mathrm{mL}, 300 \mu \mathrm{g} / \mathrm{mL}, 200 \mu \mathrm{g} / \mathrm{mL}, 150 \mu \mathrm{g} / \mathrm{mL}, 100 \mu \mathrm{g} /$ $\mathrm{mL}$, and $50 \mu \mathrm{g} / \mathrm{mL}$ ) to determine an optimal concentration that would promote colistin diffusion in agar, but not inhibit bacterial growth. Protamine was measured, mixed with distilled water until completely dissolved, and then added to the MHA before autoclaving.

\section{Phase II}

Colistin MIC and inhibition zone diameter around colistin disks on modified MHA

In total, 60 GN clinical isolates obtained from Severance hospital, including $P$. aeruginosa $(\mathrm{N}=27)$ and $\mathrm{ACB}(\mathrm{N}=33)$, were tested (Table 1 ). The study was approved by the Institutional Review Board of Yonsei University Health system, Seoul, Korea. (1-2017-0079). The clinical strains were collected from sputum and urine in 2017 and stored at $-70^{\circ} \mathrm{C}$.

Quality control was performed using E. coli (ATCC 25922), P. aeruginosa (ATCC 27853), and mcr-1-harboring $K$. aerogenes (a clinical isolate). Colistin MICs were determined by BMD for all 60 strains using colistin sulfate salt (Sigma-Aldrich, St. Louis, MO, USA) and polystyrene 96-well microplates (Corning, NY, USA). As shown in Table 1, disk diffusion AST results were interpreted based on the breakpoints, which showed good agreement with the MIC determined by BMD according to the 2018 CLSI guidelines [21]. Each strain was tested using predetermined media, i.e., commercial MHA (100\% agar concentration), MHA with 30\% agar (MHA30), and MHA30 with $100 \mu \mathrm{g} / \mathrm{mL}$ protamine (MHA30P100), which were selected based on the optimization in phase I. The colistin disk diffusion test was performed using a $10 \mathrm{mg}$ colistin disk on MHA plates that were incubated 
Table 1. Comparison between colistin MICs and inhibition zone diameters for $P$. aeruginosa and ACB complex

\begin{tabular}{|c|c|c|c|c|c|c|c|c|c|}
\hline \multirow{3}{*}{\multicolumn{2}{|c|}{ Inhibition zone diameter (mm) with: }} & \multicolumn{4}{|c|}{ P. aeruginosa isolates with colistin MIC ( $\mu \mathrm{g} / \mathrm{mL})(\mathrm{N})$} & \multicolumn{4}{|c|}{ ACB isolates with colistin MIC ( $\mu \mathrm{g} / \mathrm{mL})(\mathrm{N})$} \\
\hline & & \multicolumn{2}{|c|}{ Susceptible } & \multicolumn{2}{|c|}{ Resistant } & \multicolumn{2}{|c|}{ Susceptible } & \multicolumn{2}{|c|}{ Resistant } \\
\hline & & 1 & 2 & 4 & 8 & 1 & 2 & 4 & 8 \\
\hline \multicolumn{10}{|l|}{ MHA } \\
\hline \multirow[t]{5}{*}{ Resistant } & 6 & & & & 1 & & & & 11 \\
\hline & 7 & & & & & & & & \\
\hline & 8 & & & & 2 & & & & 2 \\
\hline & 9 & & & & & & & & \\
\hline & 10 & & & & & & 2 & 1 & 1 \\
\hline \multirow[t]{8}{*}{ Susceptible } & 11 & 1 & 10 & & & 1 & 12 & & 1 \\
\hline & 12 & 1 & 10 & & & 1 & 1 & & \\
\hline & 13 & & 2 & & & & & & \\
\hline & 14 & & & & & & & & \\
\hline & 15 & & & & & & & & \\
\hline & 16 & & & & & & & & \\
\hline & 17 & & & & & & & & \\
\hline & 18 & & & & & & & & \\
\hline \multicolumn{10}{|l|}{ МНАЗО } \\
\hline \multirow[t]{7}{*}{ Resistant } & 6 & & & & 1 & & & & 5 \\
\hline & 7 & & & & & & & & \\
\hline & 8 & & & & 2 & & & & 8 \\
\hline & 9 & & & & & & & & \\
\hline & 10 & & & & & & & 1 & \\
\hline & 11 & & & & & & & & 1 \\
\hline & 12 & & & & & & & & \\
\hline \multirow[t]{6}{*}{ Susceptible } & 13 & & & & & & 3 & & \\
\hline & 14 & 1 & 5 & & & 1 & 10 & & 1 \\
\hline & 15 & 1 & 14 & & & 1 & 2 & & \\
\hline & 16 & & 3 & & & & & & \\
\hline & 17 & & & & & & & & \\
\hline & 18 & & & & & & & & \\
\hline \multicolumn{10}{|l|}{ MHA30P100 } \\
\hline \multirow[t]{8}{*}{ Resistant } & 6 & & & & 1 & & & & 6 \\
\hline & 7 & & & & & & & & \\
\hline & 8 & & & & 2 & & & & 6 \\
\hline & 9 & & & & & & & & \\
\hline & 10 & & & & & & & & 2 \\
\hline & 11 & & & & & & & 1 & \\
\hline & 12 & & & & & & & & \\
\hline & 13 & & & & & & & & \\
\hline \multirow[t]{5}{*}{ Susceptible } & 14 & & & & & & & & \\
\hline & 15 & & 1 & & & & 7 & & 1 \\
\hline & 16 & 2 & 7 & & & 1 & 8 & & \\
\hline & 17 & & 11 & & & & & & \\
\hline & 18 & & 3 & & & 1 & & & \\
\hline
\end{tabular}

Abbreviations: ACB, Acinetobacter calcoaceticus-baumannii complex; MHA, Mueller-Hinton agar with 100\% agar concentration; MHA30, MHA with 30\% agar concentration; MHA30P100, MHA30 with 100 gg/mL protamine; MIC, minimum inhibitory concentration; R, resistance; S, Susceptible. 
at $35^{\circ} \mathrm{C}$ for $16-18$ hours in $5 \% \mathrm{CO}_{2}$.

\section{Statistical analysis}

Descriptive and statistical analyses were performed using SPSS 21 (Armonk, NY, USA) and MedCalc Statistical Software 18.10 (MedCalc Software, Ostend, Belgium; http://www.medcalc.org). Colistin MICs, as reference test, and disk diffusion results were compared to calculate sensitivity, specificity, and Kappa value of this simple disk diffusion test. Area under the curve (AUC) for inhibition zone diameters cutoff was determined from the receiver operating characteristic curve. $P<0.05$ was considered statistically significant.

\section{RESULTS}

\section{Phase I}

Colistin diffusion was optimized using MHA30, because we could not use the MHA with $20 \%$ or $10 \%$ agar (data not shown). At $30 \%$ agar concentration, the addition of protamine inhibited bacterial growth and enhanced colistin diffusion. Colistin diffusion in the agar improved with protamine supplementation and a reduction in the agar concentration Su. Inhibition zone diameters on MHA30P100 and MHA30 supplemented with $150 \mu \mathrm{g} /$ $\mathrm{mL}$ of protamine (MHA30P150) were similar for all bacterial iso-

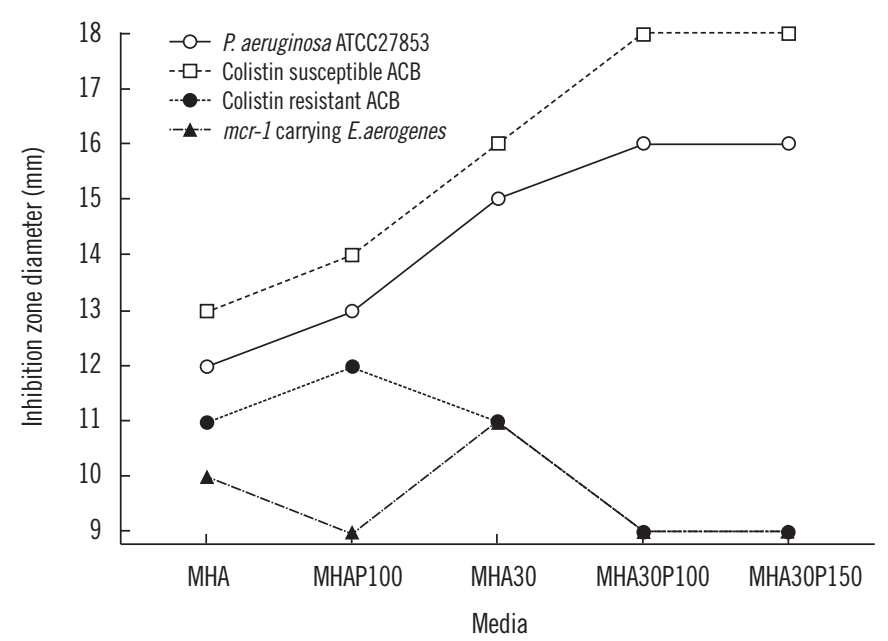

Fig. 1. Change in the colistin inhibition zone diameters with commercial MHA (MHA), commercial MHA with $100 \mu \mathrm{g} / \mathrm{mL}$ protamine (MHAP100), MHA with 30\% agar (MHA30), MHA30 with $100 \mu \mathrm{g} /$ $\mathrm{mL}$ protamine (MHA30P100), and MHA30 with $150 \mu \mathrm{g} / \mathrm{mL}$ protamine (MHA30P150). The diffusion of colistin was improved by reducing agar concentration and protamine addition. The difference in inhibition zone diameter $(\mathrm{mm})$ around colistin disks between resistant and susceptible strains became apparent in MHA30P100 and MHA30P150. lates tested. MHA30P100 was determined as the optimal medium because bacterial growth was hampered at higher protamine concentrations (Supplemental Data Table S1).

None of the strains grew on MHA30 with $\geq 300 \mu \mathrm{g} / \mathrm{mL}$ of protamine. However, colistin-susceptible ACB strain, $P$. aeruginosa ATCC 27853, and $K$. aerogenes strains grew well on MHA30 supplemented with $200 \mu \mathrm{g} / \mathrm{mL}$ of protamine, whereas colistinresistant $\mathrm{ACB}$ strains did not. Both $P$. aeruginosa and $\mathrm{ACB}$ strains grew well on MHA30P150 (Supplemental Data Table S1). Inhibition zone diameters differed between MHA30P100 and MHA30P150 ( $P<0.001)$ (Fig. 1). Hence, we decide to adopt MHA$30 \mathrm{P} 100$.

\section{Phase II}

We could not discriminate between colistin-susceptible and -resistant strains using commercial MHA. The categorical agreements between MICs determined by BMD were $100 \%$ in P. aeruginosa (27/27) and 97\% in ACB (32/33), when bacteria were grown on MHA30P100 (Table 1). Inhibition zone diameters were larger on MHA3OP100 than on MHA and MHA30 (Table 2, Fig. 1). The overall agreement between MICs and MHA3OP100 inhibition zone diameters was excellent, with a kappa value of 0.961 (95\% confidential interval (Cl), 0.885-1.0, $P<0.001$ ). The agreement was $100 \%$ for $P$. aeruginosa and colistin-susceptible ACB isolates, whereas it was $94.7 \%$ (18/19) for colistin-resistant isolates. A strain of Acinetobacter nosocomialis was susceptible in disk diffusion testing using MAH30P100, but resistant in BMD testing (Table 2).

The sensitivity and specificity of colistin disk diffusion testing by using MHA30P100 were $94.7 \%$ and 100\%, respectively (Table 3). By analyzing the AUC, we found that MHA30P100 medium allowed the best discrimination between susceptibility and resistance in both $P$. aeruginosa and $A C B$ strains. The AUC of MHA30P100 was $0.995(95 \% \mathrm{Cl}, 0.982-1.0)$ which was higher than that for MHA $(0.979,95 \% \mathrm{Cl}, 0.939-1.0)$ and MHA30

Table 2. Agreement between MIC and disk diffusion of $P$. aeruginosa $(\mathrm{N}=27)$ and $\mathrm{ACB}(\mathrm{N}=33)$ isolates

\begin{tabular}{llccc}
\hline \multirow{2}{*}{$\begin{array}{l}\text { Modified Mueller- } \\
\text { Hinton agar }\end{array}$} & Interpretation & \multicolumn{2}{c}{$\begin{array}{c}\text { Isolates with colistin } \\
\text { susceptibility by MIC (N) }\end{array}$} & Total \\
\cline { 3 - 4 } & & Susceptible & Resistant & \\
\hline MHA30P100 & Susceptible & 41 & 1 & 42 \\
& Resistant & 0 & 18 & 18 \\
Total & 41 & 19 & 60 \\
\hline
\end{tabular}

Abbreviations: see Table 1. 
Table 3. Sensitivity and specificity of cutoff values for each culture medium type

\begin{tabular}{lccc}
\hline $\begin{array}{l}\text { Mueller-Hinton } \\
\text { agar }\end{array}$ & Sensitivity \% & Specificity \% & $\begin{array}{c}\text { Inhibition zone } \\
\text { diameter breakpoints } \\
(\mathrm{mm}) \text { for susceptibility }\end{array}$ \\
\hline MHA & $94.7(74.0-99.9)$ & $95.1(83.5-99.4)$ & 10 \\
MHA30 & $94.7(74.0-99.9)$ & $100(91.4-100)$ & 12 \\
MHA30P100 & $94.7(74.0-99.9)$ & $100(91.4-100)$ & 13 \\
\hline
\end{tabular}

Abbreviations: see Table 1.

\section{(0.985 95\% Cl, 0.953-1.0).}

During colistin MIC determination, three ACB strains showed discrepancies of $>8 \mu \mathrm{g} / \mathrm{mL}$ between MICs using BMD and disk diffusion, and were retested twice using glass tubes to rule out false resistance finding due to colistin binding to polystyrene wells, which has been previously reported [22, 23]. Two strains were susceptible to colistin in reference BMD methods, resulting in the same category with disk diffusion tests. However, one strain remained colistin resistant, which was retested using MHA30P100 directly from glass tubes containing $0,0.5,1,2$, 4, or 8 $\mu \mathrm{g} / \mathrm{mL}$ of colistin. The bacterial isolate was sub-cultured from each tube after MIC measurement and further identified as Acinetobacter nosocomialis belonging to ACB. The isolates identified from the $0.5-1 \mu \mathrm{g} / \mathrm{mL}$ colistin tubes were susceptible to colistin, whereas those from the $2-8 \mu \mathrm{g} / \mathrm{mL}$ colistin tubes were resistant (data not shown).

\section{DISCUSSION}

The main objective of our study was to optimize MHA for improved colistin diffusion. We first modified the agar concentration, and optimized the medium composition with protamine (Fig. 1). When protamine was added to MHA30, colistin diffusion further increased, resulting in larger inhibition zones and a clear distinction between resistant and susceptible strains (Fig. 1). The addition of protamine to MHA30 resulted in the same sensitivity and specificity, but facilitated the interpretation of the results because the differences in inhibition zone diameters around the colistin disks on MHA30P100 were increased (Fig. 1, Table 1 , and Table 3). Although protamine has bacterial growth inhibition properties [24], bacterial growth was not inhibited at 100 $\mu \mathrm{g} / \mathrm{mL}$, while colistin diffusion increased; therefore, this concentration was used in further experiments as an optimal concentration [12].

Three ACB strains were resistant in BMD tests using polystyrene plates, but susceptible in disk diffusion tests using MHA30 and MHA30 P100. When BMD tests for these three strains were repeated using glass tubes, two strains were susceptible to colistin in line with the disk diffusion test results. However, one strain was resistant. To find the reason, the bacterial isolate was sub-cultured from each tube after MIC measurement. Interestingly, the isolates identified from the $0.5-1 \mu \mathrm{g} / \mathrm{mL}$ colistin tubes were susceptible, whereas those from the $2-8 \mu \mathrm{g} / \mathrm{mL}$ colistin tubes were resistant (data not shown). These findings implicated that $A$. nosocomialis can mutate in vitro after exposure to colistin in BMD, depending on the colistin concentration.

Colistin diffusion improved in MHA30P100 and therefore, this medium can be a useful tool for detecting colistin resistance. This simple medium was easy to prepare and allowed identifying colistin-resistant isolates of both $P$. aeruginosa and ACB with $94.7 \%$ and $100 \%$ specificity, respectively. No very major or major error were detected in $P$. aeruginosa as demonstrated by the $100 \%$ agreement between the MICs and inhibition zone diameters on MHA30P100 (Table 1).

A limitation of MHA30P100 is that it is softer than commercial MHA. Therefore, it needs to be handled with caution to avoid scratches or crumpling. Some strains of $P$. aeruginosa became more mucoid when grown on 30\% agar (data not shown), and mucoid colonies preferentially receded to the inhibition zone. In this case, a reduced incubation time of 12 hours could have resulted in clear inhibition zones around colistin disks. Further evaluation on the effect of incubation time reduction on the performance of this simple disk diffusion test would be required.

In summary, this study demonstrated that reducing the agar concentration to $30 \%$ of the concentration in commercial MHA dramatically improved colistin diffusion and resulted in reliable colistin susceptibility testing. This modified MHA is expected to be useful in clinical microbiology laboratories for colistin susceptibility testing.

\section{ACKNOWLEDGEMENTS}

We appreciate Myungsook Kim and Min-Hee Son for their excellent support for this experiment.

\section{AUTHOR CONTRIBUTIONS}

JDU, JHB, and DY planned the study; DK and HL had participated this study with support the experiments. JDU, JHB, and DY wrote this manuscript. 


\section{CONFLICTS OF INTEREST}

None.

\section{RESEARCH FUNDING}

This work was supported by a grant of the Korea Health Technology R\&D Project through the Korea Health Industry Development Institute, funded by the Ministry of Health \& Welfare, Korea (grant number: HI14C1324); by the Research Program funded by the Korea Centers for Disease Control and Prevention, Ministry of Health and Welfare, Korea (2019-ER5403-00); by Korea Institute of Planning and Evaluation for Technology in Food, Agriculture, Forestry and Fisheries through Agricultural Microbiome R\&D Program, funded by Ministry of Agriculture, Food and Rural Affairs (918003-4). The funders had no role in study design, data collection and analysis, decision to publish, or preparation of the manuscript.

\section{ORCID}

Jean Damascene Uwizeyimana https://orcid.org/0000-00026582-1497

Daewon Kim https://orcid.org/0000-0002-3487-5943

Hyunsook Lee Jung-Hyun Byun https://orcid.org/0000-0002-0179-9999

Dongeun Yong https://orcid.org/0000-0001-5909-5807 https://orcid.org/0000-0002-1225-8477

\section{REFERENCES}

1. Bergen PJ, Li J, Rayner CR, Nation RL. Colistin methanesulfonate is an inactive prodrug of colistin against Pseudomonas aeruginosa. Antimicrob Agents Chemother 2006;50:1953-8.

2. Stojanoski V, Sankaran B, Prasad BV, Poirel L, Nordmann P, Palzkill T. Structure of the catalytic domain of the colistin resistance enzyme MCR1. BMC Biol 2016;14:81.

3. Falagas ME and Kasiakou SK. Colistin: the revival of polymyxins for the management of multidrug-resistant gram-negative bacterial infections. Clin Infect Dis 2005:40:1333-41.

4. Gupta S, Govil D, Kakar PN, Prakash O, Arora D, Das S, et al. Colistin and polymyxin B: a re-emergence. Indian J Crit Care Med 2009;13:4953.

5. Huang J, Tang YQ, Sun JY. Intravenous colistin sulfate: a rarely used form of polymyxin $E$ for the treatment of severe multidrug-resistant Gramnegative bacterial infections. Scand J Infect Dis 2010;42:260-5.

6. Bosso JA, Liptak CA, Seilheimer DK, Harrison GM. Toxicity of colistin in cystic fibrosis patients. DICP 1991;25:1168-70.
7. Poirel L, Jayol A, Nordmann P. Polymyxins: antibacterial activity, susceptibility testing, and resistance mechanisms encoded by plasmids or chromosomes. Clin Microbiol Rev 2017;30:557-96.

8. Suzuki T, Hayashi K, Fujikawa K, Tsukamoto K. The chemical structure of polymyxin $\mathrm{E}$ : the identities of polymyxin $\mathrm{E} 1$ with colistin $\mathrm{A}$ and of polymyxin E2 with colistin B. J Biochem 1965;57:226-7.

9. Walkty A, DeCorby M, Nichol K, Karlowsky JA, Hoban DJ, Zhanel GG. In vitro activity of colistin (polymyxin E) against 3,480 isolates of gramnegative bacilli obtained from patients in Canadian hospitals in the CANWARD Study, 2007-2008. Antimicrob Agents Chemother 2009;53:4924-6.

10. Yu Z, Qin W, Lin J, Fang S, Qiu J. Antibacterial mechanisms of polymyxin and bacterial resistance. Biomed Res Int 2015;2015:679109.

11. Bialvaei AZ and Samadi Kafil H. Colistin, mechanisms and prevalence of resistance. Curr Med Res Opin 2015;31:707-21.

12. de Louvois J. Factors influencing the assay of antimicrobial drugs in clinical samples by the agar plate diffusion method. J Antimicrob Chemother 1982;9:253-65.

13. Dafopoulou K, Zarkotou O, Dimitroulia E, Hadjichristodoulou C, Gennimata V, Pournaras S, et al. Comparative evaluation of colistin susceptibility testing methods among carbapenem-nonsusceptible Klebsiella pneumoniae and Acinetobacter baumannii clinical isolates. Antimicrob Agents Chemother 2015;59:4625-30.

14. Balaji V, Jeremiah S, Baliga P. Polymyxins: antimicrobial susceptibility concerns and therapeutic options. Indian J Med Microbiol 2011;29:23042.

15. Galani I, Kontopidou F, Souli M, Rekatsina PD, Koratzanis E, Deliolanis J, et al. Colistin susceptibility testing by Etest and disk diffusion methods. Int J Antimicrob Agents 2008;31:434-9.

16. Moskowitz SM, Ernst RK, Miller SI. PmrAB, a two-component regulatory system of Pseudomonas aeruginosa that modulates resistance to cationic antimicrobial peptides and addition of aminoarabinose to lipid A. J Bacteriol 2004;186:575-9.

17. Moskowitz SM, Garber E, Chen Y, Clock SA, Tabibi S, Miller AK, et al. Colistin susceptibility testing: evaluation of reliability for cystic fibrosis isolates of Pseudomonas aeruginosa and Stenotrophomonas maltophilia. J Antimicrob Chemother 2010;65:1416-23.

18. Simar S, Sibley D, Ashcraft D, Pankey G. Colistin and polymyxin B minimal inhibitory concentrations determined by Etest found unreliable for gram-negative bacilli. Ochsner J 2017;17:239-42.

19. Tan T and Ng S. Comparison of Etest, Vitek and agar dilution for susceptibility testing of colistin. Clin Microbiol Infect 2007;13:541-4.

20. Bakthavatchalam YD and Veeraraghavan B. Challenges, issues and warnings from CLSI and EUCAST working group on Polymyxin susceptibility testing. J Clin Diagn Res 2017;11:DL03-DL4.

21. CLSI. Performance standards for antimicrobial susceptibility testing. 28th ed. CLSI supplement M100. Wayne, PA: Clinical and Laboratory Standards Institute, 2018.

22. Humphries RM. Susceptibility testing of the polymyxins: where are we now? Pharmacotherapy 2015;35:22-7.

23. Turlej-Rogacka A, Xavier BB, Janssens L, Lammens C, Zarkotou O, Pournaras $\mathrm{S}$, et al. Evaluation of colistin stability in agar and comparison of four methods for MIC testing of colistin. Eur J Clin Microbiol Infect Dis 2018;37:345-53.

24. Uyttendaele M and Debevere J. Evaluation of the antimicrobial activity of protamine. Food Microbiol 1994;11:417-27. 